\title{
Carcinoid and Other Neuroendocrine Tumors of the Colon and Rectum
}

\author{
John Carl Eggenberger, M.D. ${ }^{1,2}$
}

\section{ABSTRACT}

Neuroendocrine tumors (NETs) are found throughout the intestinal tract and arise from the Kulchitsky cells located in the crypts of Lieberkühn. They are classified by site of origin and by degree of differentiation, with well-differentiated lesions representing those tumors formerly referred to as carcinoid tumors. The focus of this review is NETs of the appendix, colon, and rectum. The clinical presentation of these tumors is dependent on the primary site and many are discovered incidentally, either during screening or during the investigation of nonspecific abdominal complaints. Treatment is primarily via surgical removal as the response to chemotherapy has been traditionally poor. A noted exception to this has been with treatment of the carcinoid syndrome, which occurs almost exclusively in patients with liver metastases and is due to the release of bioactive peptides and amines directly into the systemic circulation. The use of somatostatin congeners to block the release of these substances has greatly ameliorated the devastating symptoms of this condition. Postresection follow-up is advocated, but specific recommendations are lacking an evidentiary basis. NETS, particularly those of the small bowel, colon, and appendix, are seen in association with other synchronous or metachronous malignancies, often of the gastrointestinal tract. However, the utility of subsequent screening and surveillance is unproven.

KEYWORDS: NETs, carcinoid tumor, carcinoid syndrome, serotonin, somatostatin

Objectives: Upon completion of this article, the reader should be able to recognize the clinical presentation of neuroendocrine tumors (NETs) and carcinoid tumors of the appendix, colon, and rectum, as well as summarize both the surgical and medical approaches to treatment based on tumor characteristics, site of origin, and tumor stage. In addition, the reader should be able to summarize: (1) the manifestations and treatment of the carcinoid syndrome and the prevention of carcinoid crisis; and (2) the follow-up schema for patients with both localized and disseminated NETs following initial surgical treatment.

Although Langhans was the first to describe an intestinal carcinoid tumor in $1867,{ }^{1}$ it was Lubarsch who described its histologic features and classified it as a carcinoma in $1888 .^{2}$ The term carcinoid ("karzinoide" or carcinoma-like) was coined in 1907 by Oberndorfer, who felt that its more benign biologic behavior served to distinguish it from the common carcinomas of the gastrointestinal (GI) tract. $^{3}$ The relationship of intestinal carcinoids to the endocrine system was not appreciated until 1914, when it was described by Gosset and Mason. ${ }^{4}$

In 1963, Williams and Sandler proposed the first classification system for intestinal carcinoids, basing it on
${ }^{1}$ Department of Surgery, St. Joseph Mercy Hospital, Ann Arbor, Michigan; ${ }^{2}$ Associates in General and Vascular Surgery, Ypsilanti, Michigan.

Address for correspondence and reprint requests: John Carl Eggenberger, M.D., Associates in General and Vascular Surgery, Michigan Heart and Vascular Institute, 5325 Elliott Dr., Ste. 104, Ypsilanti, MI 48197 (e-mail: eggenbej@trinity-health.org).
Uncommon Colorectal Neoplasms; Guest Editor, Craig A. Reickert, M.D.

Clin Colon Rectal Surg 2011;24:129-134. Copyright (C) 2011 by Thieme Medical Publishers, Inc., 333 Seventh Avenue, New York, NY 10001, USA. Tel: $+1(212)$ 584-4662.

DOI: http://dx.doi.org/10.1055/s-0031-1285996.

ISSN 1531-0043. 
the embryologic site of origin of the tumor. ${ }^{5}$ This classification into foregut, midgut, and hindgut-derived tumors emphasized the clinical and pathologic differences between the groups, but did not address the biochemical differences between or within each category. The World Health Organization (WHO) in 2000 revised an earlier classification system to recognize both the growing heterogeneity of these tumors as well as their neuroendocrine origin. ${ }^{6}$ All lesions are termed neuroendocrine tumors (heretofore referred to as "NETs") and are separated on the basis of histologic aggressiveness and location within the GI tract. Grade 1 NETs are considered low-grade malignancies $(<2 \mathrm{mi}-$ toses per high-powered field [HPF] ), grade 2 NETs are considered to be intermediate in behavior (2-20 mitoses/ $\mathrm{HPF})$, and grade 3 are considered high grade $(>20$ mitoses/HPF). The original term "carcinoid" equates to NETs that are both grade 1 and 2 (well differentiated), whereas grade $3 \mathrm{NET}$ s are mostly small cell carcinomas with a minority component being large cell type (poorly differentiated). Finally, the focus of this review, neuroendocrine tumors of the colon and rectum, comprise only a portion of the larger group of intestinal NETs, commonly referred to as gastroenteropancreatic neuroendocrine tumors or GEP-NETs.

Neuroendocrine tumors of the colon and rectum are derived from the so-called Kulchitsky or enterochromaffin cells of the gut, found throughout the intestinal tract within the crypts of Lieberkühn. These cells have historically been referred to as APUD (amine precursor uptake and decarboxylation) cells and their related tumors as APUDomas. They stain positively when exposed to silver-based reagents and markers of neural tissue such as neuron-specific enolase and synaptophysin. Electron microscopy has demonstrated these precursor cells to contain membrane-bound secretory granules, which have been found to contain over 40 different hormones and biogenic amines. ${ }^{7}$ Elaborated substances include norepinephrine, dopamine, histamine, vasoactive intestinal peptide (VIP), substance $\mathrm{P}$, adrenocorticotropic hormone (ACTH), growth hormone, glucagon, pancreatic polypeptide (PP), gastrin, and serotonin (which will be addressed in more detail in the section on carcinoid syndrome).

\section{PRESENTATION AND DIAGNOSIS}

The clinical presentation of NETs can be nonspecific and often depends on the site of origin. Many NETs are found incidentally or in the asymptomatic patient undergoing screening or evaluative testing for unrelated reasons. Symptoms when present can be due to tumor mass effect, tumor-specific fibrosis/desmoplastic reaction, or the effects of biologically active substances secreted by the tumor. Abdominal imaging (computed tomography [CT] or magnetic resonance imaging [MRI]) combined with lower endoscopy most often provides sufficient information to make the diagnosis of and stage a colorectal NET. Small bowel series, octreotide scintigraphy, metaiodobenzylguanidine (MIBG) scintigraphy, and positron emission tomography (PET) scanning all have been used to improve the preoperative diagnostic and staging accuracy of these patients. Because of the varied presentations, I will address each tumor site individually.

\section{Appendiceal NETs}

Neuroendocrine cells of the appendix are most numerous at the tip; this may explain why most appendiceal NETs are discovered incidentally during surgery or on radiographic evaluation performed for other conditions, such as appendicitis, gynecologic symptoms, or nonspecific abdominal complaints. Few patients actually present with classic signs and symptoms of acute appendicitis and rarer still, are those who present with the carcinoid syndrome.

\section{Colonic NETs}

The majority of colonic NETs are found in the cecum or the ascending colon, although it may be difficult to differentiate between those arising from the base of the appendix and cecum. Most right-sided colonic NETs present late due to the increased diameter of the cecum and ascending colon compared with that of the descending and sigmoid colons. Thus, upwards of two-thirds are metastatic at the time of diagnosis. ${ }^{8,9}$ Although occasionally discovered during screening colonoscopy, most colonic NETs present with symptoms including bleeding, abdominal pain, change in bowel habits, anorexia, weight loss, and weakness. The carcinoid syndrome is uncommon.

\section{Rectal NETs}

About half of all rectal NETs are diagnosed at the time of routine lower endoscopy, done either for screening purposes or to evaluate unrelated symptoms. ${ }^{10-12}$ Symptoms are similar to those of patients with rectal adenocarcinoma and include bleeding of a bright red nature, pelvic/rectal discomfort, and a change in bowel habits. The carcinoid syndrome is rarely seen with rectal NETs.

\section{TREATMENT}

Surgery offers the best prognosis for all midgut and hindgut NETs. An attempt should be made to resect all visible primary disease. In the case of an advanced tumor not amenable to complete resection, tumor debulking can reduce symptoms and prevent subsequent complications. There is insufficient evidence to recommend adjuvant therapy following curative-intent surgery for locoregional disease. ${ }^{13,14}$ 


\section{Appendiceal NETs}

As most NETs of the appendix are discovered incidentally following appendectomy performed for suspected acute appendicitis, definitive management must occur postoperatively. Recall that $90 \%$ of appendiceal NETs are found at the tip. Tumors $<2 \mathrm{~cm}$ in size rarely metastasize unless there is evidence of mesoappendiceal or lymphovascular invasion. Therefore, tumors $<2 \mathrm{~cm}$ without mesoappendiceal or lymphovascular involvement can be treated with simple appendectomy. ${ }^{13} \mathrm{On}^{-}$ cologic right colectomy should be performed for those lesions with invasion at the appendiceal base, those $>2 \mathrm{~cm}$ in size, those where size cannot be confirmed, cases of incomplete resection, those lesions with evidence of lymphovascular invasion or invasion of the mesoappendix, patients with intermediate or high-grade tumors, or those with mixed histology (goblet cell carcinoma and adenocarcinoid, both of which are biologically more aggressive than their low-grade carcinoid relatives). ${ }^{13}$

\section{Colonic NETs}

NETs of the cecum and ascending colon are more common than those occurring in the transverse and descending colons. However, colonic NETs as a group tend to present late as large $(>2 \mathrm{~cm})$ masses with regional lymph node metastases. Treatment is the same as for colonic adenocarcinoma with segmental colectomy to include wide regional lymphadenectomy. ${ }^{13,14}$

\section{Rectal NETs}

Most rectal NETs are small $(<1-2 \mathrm{~cm})$ and submucosal. Such tumors rarely metastasize. Therefore, either endoscopic or surgical techniques should be employed to ensure negative resection margins. Conventional snare polypectomy or two-channel forceps-assisted snare polypectomy will achieve negative margins in the vast majority of such cases. For those with positive margins, either repeat snare polypectomy (facilitated by tattooing of the site) or transanal surgical resection should be performed to ensure complete tumor removal. ${ }^{12,14}$

Tumors that are more broadly based or closer to $2 \mathrm{~cm}$ in size do not lend themselves easily to endoscopic resection. Therefore, these tumors are usually approached via conventional transanal resection or transanal endoscopic microsurgery (TEM), a minimally invasive technique analogous to rectal laparoscopy. ${ }^{12,14}$

Tumors $>2 \mathrm{~cm}$, those invading the muscularis propria, or those with locoregional lymph node metastases are managed as per rectal adenocarcinoma (with the exception of neoadjuvant chemoradiation) with anterior, low anterior or abdominoperineal resection, depending on the distance from the pelvic floor. ${ }^{12,14}$
Transrectal ultrasound can help differentiate those tumors amenable to local therapy from those requiring radical resection by demonstrating the presence or absence of muscular invasion and lymphadenopathy.

\section{Carcinoid Syndrome}

The carcinoid syndrome is a constellation of symptoms consisting of cutaneous flushing, diarrhea, wheezing, and hemodynamic instability, caused by the systemic effects of substances elaborated by hepatic metastases. Primary NETs of the bronchi and ovary can cause the syndrome without metastatic disease. Long-term effects can result in carcinoid cardiac disease (affecting the tricuspid and pulmonary valves) and pellagra (diarrhea, dementia, dermatitis) due to niacin deficiency. Patients can present prior-or subsequent-to surgery performed to address the primary NET.

Carcinoid syndrome symptoms should prompt an imaging and biochemical workup to determine location, extent, and degree of metastatic disease. Baseline and serial urinary 5-HIAA levels allow for assessment of treatment response. A variety of imaging modalities exist for the assessment of metastatic NETs. The NANETs Consensus Guidelines recommend posteroanterior/lateral chest $\mathrm{x}$-ray combined with $\mathrm{CT}$ or MRI of the abdomen/pelvis and ${ }^{111} \mathrm{In}-\mathrm{DTPA}^{0}$ octreotide scintigraphy. This latter test takes advantage of the binding to tumor somatostatin receptors 2 and 5 and is $80-90 \%$ sensitive for both primary and metastatic NETS. ${ }^{15}$

Management of the carcinoid syndrome is considered separate from that of metastatic disease, as some patients will develop metastatic NETs without biochemical functionality. The management of the symptoms of the carcinoid syndrome has vastly improved with the introduction of synthetic somatostatin analogs (USAoctreotide, Europe-lanreotide). Octreotide is available in both an immediate and sustained-release formula (LAR) and is the mainstay of treatment. Octreotide LAR is given as a monthly intramuscular injection and may take 3 to 4 months to reach effective steady-state levels. It can be supplemented with the short-acting octreotide, which is administered as a subcutaneous (SQ) injection three times daily. Side effects of both formulations include nausea, abdominal pain, bloating, loose stools, and cholestasis/gallstones.

Other strategies to control carcinoid syndrome symptoms include the avoidance of predisposing factors (alcohol, physical activity causing pressure to the liver), antidiarrheals (Imodium, Lomotil, cholestyramine, opiates, cyproheptadine - a serotonin antagonist), and use of medications to control bronchospasm (albuterol, theophylline).

Interferon $\alpha 2 \mathrm{~b}$ and $2 \mathrm{a}$ have been used to control refractory symptoms, especially flushing and diarrhea. 
NETS contain specific interferon receptors that activate tumor suppressor genes leading to symptomatic improvement in 30 to $70 \%$ of patients. ${ }^{13}$ Side effects sometimes limit their use and include a flu-like syndrome, anorexia, weight loss, fatigue, fevers, chills, myalgias, and bone marrow suppression.

Traditional chemotherapy, such as 5-fluorouracil and streptozotocin have been used, but have shown minimal efficacy in several randomized trials. ${ }^{13}$

Surgery has a limited role in the management of the carcinoid syndrome as most of these patients have extensive disease. However, in select cases, resection of limited hepatic, nodal, or cerebral metastases has provided significant palliation of symptoms. Minimally invasive techniques have also proven beneficial in select patients for the treatment of symptomatic hepatic disease and include hepatic artery embolization, chemoembolization, radioembolization, radiofrequency ablation, and cryoablation. Experience with orthotopic liver transplantation is extremely limited and currently is considered on a case-by-case basis.

Carcinoid crisis deserves special mention. This condition, which manifests as hemodynamic instability (hypotension or hypertension), flushing, tachycardia or bradycardia, bronchospasm or complete vasomotor collapse, can be triggered by even minor surgical procedures. ${ }^{16}$ It is best prevented rather than treated. Pretreatment with octreotide in a dose of 250-500 $\mu \mathrm{g}$ SQ prior to minor procedures should suffice. ${ }^{17}$ Additional drug should be immediately available. For major procedures, a preoperative SQ dose of $250-500 \mu \mathrm{g}$ is given, followed by a continuous infusion of $50-500 \mu \mathrm{g} / \mathrm{h}$, then weaned by $50 \%$ each day until the patient no longer manifests symptoms is recommended. ${ }^{16,18,19}$ Short-acting steroid preparations and antihistamines have been recommended as adjuncts to octreotide by some authors. ${ }^{20}$ Pressor agents should be avoided, as they are associated with the release of serotonin and other vasoactive amines from NETs. ${ }^{16}$

\section{Metastatic Disease}

The lack of a standardized approach to metastatic NETs is due in large part to the paucity of data on treatment outcomes. The observation that octreotide therapy exerts both an antisecretory and antiproliferative effect on metastatic hormonally active NETs has led investigators to use it in patients with all types of metastatic disease. Although shown to delay time to tumor progression, there is currently no evidence of improved survival in such patients. ${ }^{21}$

Interferon $\alpha$, like octreotide, has demonstrated antisecretory and antiproliferative effects on metastatic NETs. However, the data is insufficient to recommend its routine use in these patients. ${ }^{22}$ It may eventually find a role in the management of octreotide-refractory metastatic NETs.

Hepatic arterial embolization and chemoembolization take advantage of hepatic physiology. Metastatic tumors derive their blood supply from branches of the hepatic arteries, while liver parenchyma is supplied mostly from the portal vein. Radiographic response rates of up to $50 \%$ have been achieved in patients with symptomatic or enlarging hepatic tumors. ${ }^{23-26}$ Data are lacking on the long-term results, however, of this form of therapy.

Cytoreductive techniques, such as radiofrequency ablation or cryoablation are utilized frequently in patients with all types of hepatic metastases, especially in patients with limited, but bilobar disease. Although applied to patients with metastatic colorectal NETS, there are no data with regard to outcome.

Conventional chemotherapy has been utilized and demonstrates variable response rates in patients with metastatic NETs. A variety of drugs has been used, including 5-fluorouracil, streptozocin, doxorubicin, capecitabine, and temozolomide. The combination of significant side effects and lack of outcome data limit the use of such agents to those patients who remain symptomatic after failing other more conventional therapy.

The use of radiolabeled somatostatin analogs in limited European trials has demonstrated response rates of $30 \%$ in patients with receptor positive metastatic NETs. ${ }^{27}$ However, the overall lack of evidence supporting any particular type of treatment for metastatic NETs argues strongly for entry of such patients into multiinstitutional clinical trials. ${ }^{14}$

\section{RESULTS}

Regardless of primary site, the survival of patients with GI tract NETs parallels the stage of the tumor. Additionally, presence of the carcinoid syndrome in patients with metastatic disease confers a poorer overall survival compared with those with nonsyndromic metastatic disease.

For appendiceal NETs, 5-year survival rates are $80.8 \%$ for localized disease, $88.1 \%$ for regional disease, and $9.6 \%$ for tumors with distant metastases. Overall, the 5 -year survival rate is $71.0 \%{ }^{28}$

For colonic NETS (excluding appendiceal tumors), 5-year survival rates are $76.0 \%$ for localized disease, $71.6 \%$ for regional disease, and $30.0 \%$ for tumors with distant metastases. Overall, the 5-year survival rate is $61.8 \% .^{28}$

The prognosis of rectal NETs falls somewhere between those of appendiceal and colonic NETs with localized tumors conveying a 5-year survival rate of $90.8 \%, 48.9 \%$ for regional tumors, and $32.2 \%$ for metastatic tumors. Overall, the 5 -year survival rate is $88.3 \%{ }^{28}$ 


\section{FOLLOW-UP}

NETs tend to be slower growing than same site adenocarcinomas and therefore may recur many years following resection. Long-term follow-up data are limited. However, it seems reasonable to exclude early, limited tumors from long-term surveillance and follow-up. These would include appendiceal NETs $<2 \mathrm{~cm}$ in size without poor prognostic factors (positive lymph nodes; angiolymphatic or mesoappendiceal invasion; intermediate, high-grade, or mixed histology) and rectal NETs that are submucosal and $<2 \mathrm{~cm}$ in diameter. ${ }^{13,14}$

All other appendiceal, colonic, and rectal NETs carry a substantial risk of recurrence. A follow-up program consisting of endoscopic and radiographic (CT or MRI) surveillance at 1- to 3-year intervals may allow for early detection, and in some instances, treatment resulting in long-term survival. Office follow-up is recommended at 6- to 12 -month intervals for a minimum of 7 years. ${ }^{13,14}$ In patients with biochemically active tumors, the monitoring of urinary 5-HIAA and serum chromogranin A can detect early recurrences. Finally, the use of surveillance octreotide scintigraphy can supplement follow-up based on individual circumstances.

The presence of GI tract NETs is associated with the concomitant or subsequent development of other neoplasms. Many are of GI tract origin, but can also include prostate, ovary, and lung. Most are seen in association with small bowel NETs, but up to $13 \%$ of rectal lesions and 18\% of appendiceal tumors demonstrate this phenomenon. ${ }^{28}$ It has been postulated that this high association between NETs and other neoplasms may be due to the trophic or mutagenic effects of some of the substances elaborated by NETs. Although it seems reasonable to evaluate the GI tract in patients following the diagnosis of an intestinal NET, the utility and efficacy of ongoing surveillance is unproven.

\section{REFERENCES}

1. Langhans T. Ueber einen drusenpolyp im ileum. Virchows Arch Pathol Anat Physiol Klin Med 1867;38:559-560

2. Lubarsch O. Uber den primaren Krebs des ileum nebst Bemerkungen uber das gleichzeitige Vorkommen von Krebs und Tuberkulose. Virchows Arch 1888;3:280-317

3. Oberndorfer S. Karzenoide tumoren des dunndarms. Franfurt Z Pathol 1907;1:426-430

4. Gosset A, Masson P. Tumeurs endocrines de l'appendice. Presse Med 1914;25:237-239

5. Williams ED, Sandler M. The classification of carcinoid tumours. Lancet 1963;1(7275):238-239

6. Solcia E, Kloppel G, Sobin LH, et al. Histological Typing of Endocrine Tumours. WHO International Histological Classification of Tumours. 2nd ed. Berlin: Springer; 2000

7. Delcore R, Friesen SR. Gastrointestinal neuroendocrine tumors. J Am Coll Surg 1994;178(2):187-211
8. Crocetti E, Paci E. Malignant carcinoids in the USA, SEER 1992-1999. An epidemiological study with 6830 cases. Eur J Cancer Prev 2003;12(3):191-194

9. Kulke MH, Mayer RJ. Carcinoid tumors. N Engl J Med 1999; 340(11):858-868

10. Shields CJ, Tiret E, Winter DC; International Rectal Carcinoid Study Group. Carcinoid tumors of the rectum: a multi-institutional international collaboration. Ann Surg 2010;252(5):750-755

11. Yoon SN, Yu CS, Shin US, Kim CW, Lim SB, Kim JC. Clinicopathological characteristics of rectal carcinoids. Int J Colorectal Dis 2010;25(9):1087-1092

12. Kwaan MR, Goldberg JE, Bleday R. Rectal carcinoid tumors: review of results after endoscopic and surgical therapy. Arch Surg 2008;143(5):471-475

13. Boudreaux JP, Klimstra DS, Hassan MM, et al; North American Neuroendocrine Tumor Society (NANETS). The NANETS consensus guideline for the diagnosis and management of neuroendocrine tumors: well-differentiated neuroendocrine tumors of the jejunum, ileum, appendix, and cecum. Pancreas 2010;39(6):753-766

14. Anthony LB, Strosberg JR, Klimstra DS, et al; North American Neuroendocrine Tumor Society (NANETS). The NANETS consensus guidelines for the diagnosis and management of gastrointestinal neuroendocrine tumors (NETS): welldifferentiated NETS of the distal colon and rectum. Pancreas 2010;39(6):767-774

15. Krenning EP, Kooij PPM, Bakker WH, et al. Radiotherapy with a radiolabeled somatostatin analogue, [111In-DTPAD-Phe1]-octreotide. A case history. Ann N Y Acad Sci 1994; 733:496-506

16. Honig LJ, Weingarten G. A gastric carcinoid tumor with massive bleeding. Am J Gastroenterol 1974;61(1):40-44

17. Parris WC, Oates JA, Kambam J, Shmerling R, Sawyers JF. Pre-treatment with somatostatin in the anaesthetic management of a patient with carcinoid syndrome. Can J Anaesth 1988;35(4):413-416

18. Quinlivan JK, Roberts WA. Intraoperative octreotide for refractory carcinoid-induced bronchospasm. Anesth Analg 1994;78(2):400-402

19. Claure RE, Drover DD, Haddow GR, Esquivel CO, Angst MS. Orthotopic liver transplantation for carcinoid tumour metastatic to the liver: anesthetic management. Can J Anaesth 2000;47(4):334-337

20. Orbach-Zinger S, Lombroso R, Eidelman LA. Uneventful spinal anesthesia for a patient with carcinoid syndrome managed with long-acting octreotide. Can J Anaesth 2002; 49(7):678-681

21. Rinke A, Müller HH, Schade-Brittinger C, et al; PROMID Study Group. Placebo-controlled, double-blind, prospective, randomized study on the effect of octreotide LAR in the control of tumor growth in patients with metastatic neuroendocrine midgut tumors: a report from the PROMID Study Group. J Clin Oncol 2009;27(28):4656-4663

22. Schnirer II, Yao JC, Ajani JA. Carcinoid-a comprehensive review. Acta Oncol 2003;42(7):672-692

23. Eriksson BK, Larsson EG, Skogseid BM, Löfberg AM, Lörelius LE, Oberg KE. Liver embolizations of patients with malignant neuroendocrine gastrointestinal tumors. Cancer 1998;83(11):2293-2301

24. Gupta S, Yao JC, Ahrar K, et al. Hepatic artery embolization and chemoembolization for treatment of patients with 
metastatic carcinoid tumors: the M.D. Anderson experience. Cancer J 2003;9(4):261-267

25. Strosberg JR, Choi J, Cantor AB, Kvols LK. Selective hepatic artery embolization for treatment of patients with metastatic carcinoid and pancreatic endocrine tumors. Cancer Contr 2006;13(1):72-78

26. Kennedy AS, Dezarn WA, McNeillie P, et al. Radioembolization for unresectable neuroendocrine hepatic metastases using resin 90Y-microspheres: early results in 148 patients. Am J Clin Oncol 2008;31(3):271-279

27. Kwekkeboom DJ, de Herder WW, Kam BL, et al. Treatment with the radiolabeled somatostatin analog $[177 \mathrm{Lu}-$ DOTA 0,Tyr3] octreotate: toxicity, efficacy, and survival. J Clin Oncol 2008;26(13):2124-2130

28. Modlin IM, Lye KD, Kidd M. A 5-decade analysis of 13,715 carcinoid tumors. Cancer 2003;97(4):934-959 